\title{
HIV incidence estimates by sex and age group in the population aged 15 years or over, Brazil, 1986-2018
}

\author{
Célia Landmann Szwarcwald[1], Paulo Roberto Borges de Souza Júnior ${ }^{[1]}$, \\ Ana Roberta Pati Pascom ${ }^{[2]}$, Ronaldo de Almeida Coelho ${ }^{[2]}$, Rachel Abrahão Ribeiro ${ }^{[2],}$ \\ Giseli Nogueira Damacena ${ }^{[1]}$, Deborah Carvalho Malta ${ }^{[3]}$, Maria Cristina Pimenta ${ }^{[2]}$ \\ and Gerson Fernandes Mendes Pereira ${ }^{[2]}$
}

[1]. Fundação Oswaldo Cruz, Instituto de Comunicação e Informação Científica e Tecnológica em Saúde, Rio de Janeiro, RJ, Brasil.
[2]. Ministério da Saúde, Departamento de Condições Crônicas e Infecções Sexualmente Transmissíveis, Brasília, DF, Brasil.

[3]. Universidade Federal de Minas Gerais, Escola de Enfermagem, Belo Horizonte, MG, Brasil.

\begin{abstract}
Introduction: HIV incidence estimates are essential to monitor the progress of prevention and control interventions. Methods: Data collected by Brazilian surveillance systems were used to derive HIV incidence estimates by age group (15-24; 25+) and sex from 1986 to 2018. This study used a back-calculation method based on the first CD4 count among treatment-naïve cases. Incidence estimates for the population aged 15 years or over were compared to Global Burden of Disease Study (GBD) estimates from 2000 to 2018. Results: Among young men (15-24 years), HIV incidence increased from 6,400 (95\% CI: 4,900-8,400), in 2000, to 12,800 (95\% CI: $10,800-$ $15,900)$, in 2015, reaching incidence rates higher than 70/100,000 inhabitants and an annual growth rate of $3.7 \%$. Among young women, HIV incidence decreased from 5,000 (95\% CI: 4,200-6,100) to 3,200 (95\% CI: 3,000-3,700). Men aged $\geq 25$ years and both female groups showed significant annual decreases in incidence rates from 2000 to 2018. In 2018, the estimated number of new infections was 48,500 (95\% CI: 45300-57500), 34,800 (95\% CI: 32800-41500) men, 13,600 (95\% CI: 12,500-16,000) women. Improvements in the time from infection to diagnosis and in the proportion of cases receiving antiretroviral therapy immediately after diagnosis were found for all groups. Comparison with GBD estimates shows similar rates for men with overlapping confidence intervals. Among women, differences are higher mainly in more recent years. Conclusions: The results indicate that efforts to control the HIV epidemic are having an impact. However, there is an urgent need to address the vulnerability of young men.
\end{abstract}

Keywords: HIV incidence. Estimation. Time trends. Young adults. GBD. Brazil.

\section{INTRODUCTION}

Over the past two decades, thanks to the effectiveness of antiretroviral therapy (ART), enormous progress has been made in improving the health and survival of HIV-infected individuals. The success of ART, combined with the benefits of treatment as prevention (TasP) and pre-exposure prophylaxis (PrEP), have generated new expectations surrounding the end of the HIV epidemic ${ }^{1}$.

To ensure early HIV diagnosis and the effective implementation of prompt access to treatment, it is essential to have HIV

\footnotetext{
Corresponding author: Célia Landmann Szwarcwald.

e-mail: celia_ls@hotmail.com

(D) https://orcid.org/0000-0002-7798-2095

Received 3 May 2021

Accepted 30 August 2021
}

incidence estimates to describe the current dynamics of the epidemic ${ }^{2}$. Particularly, if estimates can be derived by geographic areas, demographic groups, and risk categories ${ }^{3}$, trends in HIV incidence indicate not only the degree to which HIV transmission is controlled ${ }^{4}$, but also which groups are most at risk for HIV infection in the general population ${ }^{5,6}$. Despite the relevant role of HIV incidence in surveillance, estimating the annual number of new infections continues to be challenging in many countries ${ }^{7-9}$.

Historically, the calculation of HIV incidence has been based on reported AIDS cases, using back-calculation models of AIDS incidence, with the assumption that temporal trends in AIDS incidence reflect past trends in HIV incidence ${ }^{10}$. However, the expansion of antiretroviral therapy has lengthened the time to the onset of AIDS ${ }^{11}$, making inferences about HIV incidence based on AIDS reported cases limited. 
HIV incidence can also be calculated based on the change in HIV prevalence estimated at two points in time ${ }^{12}$. The assumption underlying this methodology is that the number of new infections is equal to the number of prevalent cases at the second point in time, minus the number of cases who survived between the two time points. This is the basis of the UNAIDS methodology to estimate key HIV indicators, such as the number of PLHIV, new infections, and AIDS deaths using complex mathematical models ${ }^{13}$.

In the late 1990s, laboratory tests were developed to estimate HIV incidence in cross-sectional studies ${ }^{14}$. The algorithms are based on laboratory assays that distinguish recent from long-term infections. As in cross-sectional surveys, the main advantage of this type of approach is the use of a single blood sample collected at one point in time, which does not require follow-up on subjects, as would be required in cohort studies ${ }^{5}$. Recent infection testing algorithms (RITAs), incorporating clinical information into the HIV recency assay, have proven to accurately classify recent infections and have been widely used to estimate the incidence of HIV in several countries and different epidemiological setting ${ }^{15-18}$, including HIV incidence estimation in two Brazilian cities ${ }^{19}$. However, the logistics of identifying recently acquired infections in routine testing settings may be complicated and costly for continental countries with concentrated HIV epidemics. Moreover, as there is no estimate of cumulative incidence, the biomarker method cannot be used to estimate HIV prevalence and the proportion of undiagnosed infections ${ }^{3}$.

Lately, methods based on the first CD4 count after HIV diagnosis have been developed to estimate HIV incidence in some countries ${ }^{3,6,20-25}$. The main assumption of these models is that among ART treatment-naïve individuals, CD4 cell counts decrease over time ${ }^{26}$. Although it is well-known that a small proportion of people living with HIV (PLHIV) experiences the preservation of $\mathrm{CD} 4^{27}$, these approaches have the advantage of using a CD4 count back-calculation model to estimate HIV incidence with routinely available data.

Young people are considered a key population for HIV prevention interventions in Brazil and worldwide ${ }^{28}$. Several risk factors for sexually transmitted infections (STIs) are raised amongst young people, such as being in the beginning of their sexual life, experimenting with high-risk behaviors, and feeling invulnerable ${ }^{29}$. From 2007-2019, reported HIV cases in Brazil rose by 64.6\% among young men $^{30}$. Given this epidemiological pattern, the estimation of HIV incidence among Brazilian youth seems timely.

The present study used data collected by Brazilian surveillance systems to derive HIV incidence estimates among men and women aged 15-24 years from 1986 to 2018 . Time trends of HIV incidence rates are compared to those obtained for older age groups. In addition, HIV incidence estimates from 2000 to 2018 were compared to the Global Burden of Diseases (GBD) estimates for males and females aged 15 years or over.

\section{METHODS}

In Brazil, AIDS started to be considered a compulsory notification disease in 1986. The National Information System of Notified AIDS cases (SINAN-AIDS, in Portuguese) was implemented in 1993 and has brought about important advances in terms of systematizing epidemiological data. Since 2014, the notification of HIV cases has also become mandatory, although some states had been reporting HIV cases long before this date.

In the 2000s, in addition to SINAN-AIDS, the surveillance of HIV cases started to rely on other information systems from the Ministry of Health: Mortality Information System (SIM, in Portuguese); Laboratory Examination Control System (SISCEL, in Portuguese); Logistics Control System for Medicines (SICLOM, in Portuguese); and the National System of Notified Tuberculosis cases (SINAN-TB, in Portuguese). All of these databases are linked in order to compose the integrated HIV surveillance information system (SIIHIV, in Portuguese). The integrated information system is used not only to correct the underreporting of cases in SINAN, but also for the clinical monitoring of patients.

In 2013, a model for estimating HIV incidence was proposed based on the first CD4 count among treatment-naïve patients and was applied to SISCEL/SICLOM data to monitor the number of recent infections among men and women from 2004 to $2013^{22,25}$. The approach is based on the first CD4 count after diagnosis among all treatment-naïve HIV infected cases informed to SISCEL to estimate the time since infection. To use the CD4 depletion model in Brazil, the model originally proposed by Lodi et al. ${ }^{26}$ was adapted and specific decline rates by sex and age group were estimated from Brazilian SISCEL data ${ }^{25}$.

The present study proposes an adaptation of the previously developed back-calculation method based on the first CD4 count among treatment-naïve $\operatorname{cases}^{25}$ to derive HIV incidence estimates by age-group (15-24; 25 or older) and sex using information from the integrated HIV information system (SIIHIV) from 1986 to 2018. This study was based on the Project "Estimação da incidência de HIV no Brasil utilizando sistemas de informações em saúde" ("Estimation of HIV incidence in Brazil, using health information systems"), which was approved by the Ethics Committee of the Oswaldo Cruz Foundation, Ministry of Health, Brazil (Protocol 2.134.753).

For the analysis, the first HIV detection date among all databases that compose the integrated system was assumed to be the date of HIV diagnosis. In the first step, for each treatment-naïve case of HIV infection informed to SISCEL, the Brazilian CD4 depletion model was used to estimate the time between infection and the first CD4 count, and the time between HIV infection and diagnosis. In the next step, for all cases not informed to SISCEL, the time between HIV infection and diagnosis was estimated by using a multiple imputation procedure using the information source of the first detection date and the notification criterion in SINAN (AIDS or HIV) as predictor variables.

In similarity to the previous proposal ${ }^{25}$, HIV incidence is calculated as the upper limiting value of the cumulative sum of individuals reported to SISCEL in the same year of HIV infection, in the year following the infection, two years after the infection, and so on. Under the assumption that the probability of being 
diagnosed less than $\mathrm{x}$ years after infection is expressed by a logistic probability distribution (equation 1), HIV incidence is calculated as the upper limit of the cumulative number of cases $N_{x}$ (equation 2):

$$
F(x ; \mu ; \sigma)=1 /(1+\exp (-\pi / \sqrt{3} . z)) \text { for } z=(x-\mu) / \sigma
$$

,where $\mu$ and $\sigma$ are the mean and standard deviation of time from infection to diagnosis and $x$ is the number of years after infection.

$$
N_{x}=I . F(x ; \mu ; \sigma) \text {, where } I \text { is HIV incidence. }
$$

To estimate $\mu, \sigma$, and HIV incidence $(I)$, an iterative procedure was used with an initial guess for $\sigma$ to generate successive approximations to a solution. The detailed description of the estimation method of the parameters $\mu$ and $\sigma$ can be found in a previous publication ${ }^{25}$ (Supplementary methodology section).

To estimate HIV incidence by sex and age group, age at infection was estimated as age at diagnosis minus the time between infection and diagnosis. Because HIV incidence is estimated for people aged 15 or over, if the date of infection is estimated before age 15 , it is set to the date the person reaches 15 years of age ${ }^{3}$. Using this approach, HIV incidence was estimated by sex and age group (15-24; 25+) in Brazil from 2000 to 2013 and the proportion of cases reported within the first year of HIV infection $\left(p_{1}\right)$.

As the estimated HIV incidence loses accuracy with a small number of observations ${ }^{25}$, the number of HIV cases diagnosed within the first year of infection was used to generate estimates in recent years. To estimate HIV incidence from 2014 to 2018, we first predicted the proportion of cases reported within the first year of HIV infection by fitting a regression model to the $\operatorname{logit}\left(p_{l t}\right)$ varying with time $(\mathrm{t})(\mathrm{t}=0, \ldots ., 13)$, using $\mathrm{t}, \mathrm{t}^{2}$, and $\ln (\mathrm{t})$ as independent variables and the upper limit of 0.70 :

$$
\operatorname{Ln}\left(\frac{p_{1 t}}{\left(0.70-p_{1 t}\right)}\right)=a+b_{1} \cdot t+b_{2} \cdot t^{2}+b_{3} \cdot \ln (t)
$$

The estimated coefficients of determination $\left(\mathrm{R}^{2}\right)$ were 0.96 for men aged $15-24$ years, men $\geq 25$ years old, women aged $15-24$ years, and 0.92 for women $>=25$ years.

The estimate of HIV incidence from 2014 to 2018 was then given by the ratio of the number of newly infected individuals diagnosed within the first year of HIV infection and the predicted proportion $\left(\hat{p}_{1 t}\right)$. Confidence intervals for HIV incidence were based on $95 \%$ confidence intervals $(95 \% \mathrm{CI})$ for the predicted proportions.

Incidence estimates in the Brazilian population aged 15 years or over were calculated by summing up incidence estimates by age group. Information on population by age group and sex were used to estimate HIV incidence rates. HIV incidence estimates for males and females aged 15 years or older were compared to the Global Burden of Diseases (GBD) estimates.

The software Statistical Package for the Social Sciences (IBM SPSS for Windows), version 22.0, was used for the statistical analysis.

The GBD methodology used to estimate HIV incidence in Brazil is based on cause-specific vital registration information. Mortality data were analyzed at the subnational level and were corrected for garbage coding and HIV misclassification. HIV incidence estimates were generated by back-calculation from mortality data, using assumptions of disease progression and survival. For the cohort incidence bias adjustment, a process was developed by GBD driven by Spectrum mortality and incidence cohort survival estimates. The detailed methodology is described in Murray et al. (2019) ${ }^{31}$.

\section{RESULTS}

Estimates of HIV incidence by sex and age group from 2000 to 2018 are presented in Table 1. Among young men (15-24 years), results show HIV incidence increased from 6,400 (95\% CI: 4,900$8,400)$ in 2000 to 12,800 (95\% CI: $10,800-15,900)$, in 2015 , and, after that year, began to decrease slowly. Among young women (15-24 years), results show HIV incidence decreased from 5,000 ( $95 \%$ CI: 4,200-6,100), in 2000, to 3,200 (95\% CI: 3,000-3,700), in 2018. In this age group, the male-female incidence ratio was 1.3 in 2000 and increased to 3.7 in 2018. Among men aged 15-24 years, HIV incidence rates reached values higher than 70 per 100,000 inhabitants from 2014 to 2016. Among women of the same age group, in 2018, the incidence rate was 18.6 (95\% CI:16.7-22.1) per 100,000 , almost four times smaller than among men.

In the age group of 25 years or over, men showed an increasing trend until 2015 but at a much slower pace than in the youngest age group, from $16,600(95 \%$ CI: $14,600-19,200)$ to $24,300(95 \%$ CI: 22,700-26,500), in 2015, showing a slight decrease after this year. HIV incidence among women aged 25 years or over showed little variation in the period, with approximately 11,500 new infections per year. In the oldest age group, the male-female incidence ratio increased from 1.6 to 2.2, from 2000 to 2018 . Comparing HIV incidence rates, the youngest men show the highest rates of all groups (Table 1).

Trends of HIV incidence rates by age group and sex can be examined in Figure 1. Among the youngest males, after years of pronounced growth, there is a small decline in HIV incidence rates after 2016. Among men aged 25 or over, the growth trend at the beginning of the epidemic changes to a stable level after the year 2000. Both female groups show the same pattern, a rising trend from 1986 to 2005 and a decreasing trend from 2006 to 2018, although the decrease among the youngest age group is sharper.

In 2018, the estimated number of new infections in Brazil was 48,500 (95\% CI: 45300-57500), 34,800 (95\% CI: 32800-41500) men, representing $72 \%$ of the total, and 13,600 (95\% CI: $12,500-$ 16,000 ) women. For the total population aged 15 years or over, the HIV incidence rate was 29.0 per 100,000 (95\% CI: 27.1-34.3), in 2018, with 42.9 (95\% CI: 40.3-51.0) among men and 15.8 (95\% CI: 14.6-18.6) among women. Among males, although a small but significant annual decreasing rate was found in the period 2000-2018, peak incidence rates were observed in 2014, 2015, and 2016, while for females, a continuous decrease was found after 2003 (Table 2).

Comparison with GBD estimates shows close values for men aged 15 years or over, with all confidence intervals overlapping. Small differences were found in recent years: the average ratio between the estimates was 1.06 but reaches 1.16 in 2018. Among 
TABLE 1: HIV incidence and HIV incidence rates (per 100,000 inhabitants) by sex, age group, and year of HIV infection. Brazil, 2004-18.

\begin{tabular}{|c|c|c|c|c|c|c|c|c|c|c|c|c|}
\hline \multirow{4}{*}{ Year } & \multicolumn{12}{|c|}{ Males } \\
\hline & \multicolumn{6}{|c|}{ 15-24 years } & \multicolumn{6}{|c|}{25 years or over } \\
\hline & \multirow{2}{*}{ Incidence $(x 1000)$} & \multicolumn{2}{|c|}{$95 \% \mathrm{Cl}$} & \multirow{2}{*}{$\begin{array}{c}\text { Rate } \\
(/ 100000)\end{array}$} & \multicolumn{2}{|c|}{$95 \% \mathrm{Cl}$} & \multirow{2}{*}{$\begin{array}{l}\text { Incidence } \\
(\times 1000)\end{array}$} & \multicolumn{2}{|c|}{$95 \% \mathrm{Cl}$} & \multirow{2}{*}{$\begin{array}{c}\text { Rate } \\
(/ 100000)\end{array}$} & \multicolumn{2}{|c|}{$95 \% \mathrm{Cl}$} \\
\hline & & LL & UL & & LL & UL & & LL & UL & & LL & UL \\
\hline 2000 & 6.4 & 4.9 & 8.4 & 37.7 & 28.8 & 50.0 & 16.6 & 14.6 & 19.2 & 41.3 & 36.1 & 47.7 \\
\hline 2001 & 6.7 & 5.3 & 8.4 & 39.4 & 31.5 & 49.8 & 17.4 & 15.6 & 19.5 & 42.0 & 37.7 & 47.1 \\
\hline 2002 & 7.1 & 5.7 & 8.9 & 41.7 & 33.4 & 52.7 & 17.8 & 16.0 & 20.0 & 42.0 & 37.8 & 47.1 \\
\hline 2003 & 7.5 & 6.0 & 9.4 & 44.0 & 35.4 & 55.2 & 18.3 & 16.5 & 20.5 & 42.1 & 37.9 & 47.1 \\
\hline 2004 & 7.8 & 6.3 & 9.8 & 45.8 & 37.2 & 57.1 & 18.5 & 16.7 & 20.6 & 41.5 & 37.5 & 46.3 \\
\hline 2005 & 8.0 & 6.5 & 9.9 & 46.7 & 38.0 & 57.9 & 18.7 & 16.9 & 20.9 & 41.0 & 37.1 & 45.6 \\
\hline 2006 & 8.1 & 6.6 & 10.0 & 47.1 & 38.4 & 58.4 & 19.1 & 17.3 & 21.2 & 40.7 & 36.9 & 45.3 \\
\hline 2007 & 8.2 & 6.7 & 10.2 & 47.7 & 39.1 & 59.0 & 19.5 & 17.7 & 21.7 & 40.6 & 36.9 & 45.1 \\
\hline 2008 & 8.4 & 6.9 & 10.3 & 48.6 & 39.9 & 59.8 & 19.9 & 18.1 & 22.0 & 40.4 & 36.7 & 44.7 \\
\hline 2009 & 8.6 & 7.2 & 10.6 & 49.7 & 41.2 & 60.8 & 20.2 & 18.4 & 22.2 & 39.9 & 36.5 & 43.9 \\
\hline 2010 & 9.1 & 7.6 & 11.1 & 52.5 & 43.9 & 63.6 & 20.5 & 18.8 & 22.4 & 39.5 & 36.3 & 43.2 \\
\hline 2011 & 9.9 & 8.3 & 11.8 & 56.5 & 47.8 & 67.8 & 20.8 & 19.2 & 22.6 & 39.1 & 36.2 & 42.5 \\
\hline 2012 & 10.6 & 9.0 & 12.7 & 60.6 & 51.6 & 72.5 & 21.3 & 19.8 & 23.0 & 39.0 & 36.3 & 42.2 \\
\hline 2013 & 11.6 & 9.8 & 13.9 & 65.8 & 56.0 & 79.0 & 22.4 & 20.9 & 24.2 & 40.1 & 37.4 & 43.4 \\
\hline 2014 & 12.4 & 10.5 & 15.2 & 70.6 & 59.8 & 86.1 & 23.7 & 22.2 & 25.8 & 41.4 & 38.7 & 44.9 \\
\hline 2015 & 12.8 & 10.8 & 15.9 & 72.5 & 61.1 & 90.2 & 24.3 & 22.7 & 26.5 & 41.3 & 38.6 & 45.0 \\
\hline 2016 & 12.6 & 10.7 & 16.1 & 71.3 & 60.2 & 90.6 & 24.0 & 22.5 & 26.2 & 39.8 & 37.3 & 43.5 \\
\hline 2017 & 12.0 & 10.2 & 15.5 & 67.5 & 57.6 & 87.2 & 23.2 & 21.8 & 25.3 & 37.4 & 35.3 & 40.9 \\
\hline 2018 & 11.9 & 10.9 & 16.4 & 66.5 & 61.2 & 91.8 & 23.0 & 21.9 & 25.1 & 36.2 & 34.5 & 39.5 \\
\hline \multirow{4}{*}{ Year } & \multicolumn{12}{|c|}{ Females } \\
\hline & \multicolumn{6}{|c|}{$15-24$ years } & \multicolumn{6}{|c|}{25 years or over } \\
\hline & \multirow{2}{*}{ Incidence $(x 1000)$} & \multicolumn{2}{|c|}{$95 \% \mathrm{Cl}$} & \multirow{2}{*}{$\begin{array}{c}\text { Rate } \\
(/ 100000)\end{array}$} & \multicolumn{2}{|c|}{$95 \% \mathrm{Cl}$} & Incidence & & & & & \\
\hline & & LL & UL & & & & $(x 1000)$ & LL & UL & $(/ 100000)$ & LL & UL \\
\hline 2000 & 5.0 & 4.2 & 6.1 & 29.6 & 24.9 & 35.8 & 10.7 & 9.0 & 13.0 & 24.6 & 20.7 & 30.1 \\
\hline 2001 & 5.1 & 4.5 & 5.9 & 30.3 & 26.6 & 34.9 & 11.2 & 9.8 & 13.1 & 25.3 & 22.1 & 29.5 \\
\hline 2002 & 5.1 & 4.5 & 5.8 & 29.8 & 26.4 & 34.2 & 11.7 & 10.3 & 13.6 & 25.7 & 22.6 & 29.8 \\
\hline 2003 & 5.1 & 4.5 & 5.8 & 29.9 & 26.6 & 33.9 & 12.0 & 10.6 & 13.9 & 25.7 & 22.7 & 29.7 \\
\hline 2004 & 5.0 & 4.4 & 5.6 & 29.1 & 26.1 & 32.9 & 12.1 & 10.7 & 13.9 & 25.2 & 22.3 & 28.9 \\
\hline 2005 & 4.8 & 4.4 & 5.4 & 28.4 & 25.6 & 31.9 & 12.1 & 10.7 & 13.8 & 24.5 & 21.8 & 28.0 \\
\hline 2006 & 4.6 & 4.2 & 5.2 & 27.3 & 24.6 & 30.5 & 11.9 & 10.6 & 13.6 & 23.6 & 21.0 & 26.9 \\
\hline 2007 & 4.5 & 4.1 & 5.0 & 26.4 & 23.9 & 29.5 & 11.8 & 10.6 & 13.5 & 22.8 & 20.4 & 26.0 \\
\hline 2008 & 4.4 & 4.0 & 4.9 & 25.6 & 23.3 & 28.5 & 11.8 & 10.6 & 13.4 & 22.2 & 19.9 & 25.2 \\
\hline 2009 & 4.2 & 3.9 & 4.7 & 24.7 & 22.6 & 27.4 & 11.8 & 10.6 & 13.3 & 21.6 & 19.4 & 24.3 \\
\hline 2010 & 4.1 & 3.8 & 4.5 & 23.9 & 22.0 & 26.3 & 11.7 & 10.6 & 13.1 & 20.9 & 19.0 & 23.4 \\
\hline 2011 & 4.0 & 3.7 & 4.4 & 23.3 & 21.6 & 25.5 & 11.5 & 10.5 & 12.8 & 20.1 & 18.3 & 22.3 \\
\hline 2012 & 3.9 & 3.7 & 4.3 & 23.0 & 21.3 & 25.0 & 11.4 & 10.5 & 12.7 & 19.4 & 17.8 & 21.5 \\
\hline 2013 & 3.9 & 3.6 & 4.2 & 22.6 & 21.0 & 24.7 & 11.5 & 10.6 & 12.8 & 19.1 & 17.5 & 21.2 \\
\hline 2014 & 3.9 & 3.6 & 4.3 & 22.5 & 20.9 & 24.8 & 11.6 & 10.7 & 13.0 & 18.8 & 17.2 & 21.0 \\
\hline 2015 & 3.8 & 3.5 & 4.2 & 22.0 & 20.3 & 24.4 & 11.5 & 10.5 & 13.1 & 18.1 & 16.5 & 20.6 \\
\hline 2016 & 3.6 & 3.3 & 4.0 & 20.6 & 18.9 & 23.1 & 11.0 & 10.0 & 12.7 & 16.9 & 15.4 & 19.4 \\
\hline 2017 & 3.4 & 3.1 & 3.8 & 19.5 & 17.9 & 22.2 & 10.6 & 9.7 & 12.4 & 15.8 & 14.5 & 18.5 \\
\hline 2018 & 3.2 & 3.0 & 3.7 & 18.6 & 17.2 & 21.4 & 10.4 & 9.6 & 12.3 & 15.1 & 13.9 & 17.9 \\
\hline
\end{tabular}


Rev Soc Bras Med Trap | on line | Vol.:55: | (Suppl I): e0231-2021 | 2022

Age group

$15-24$
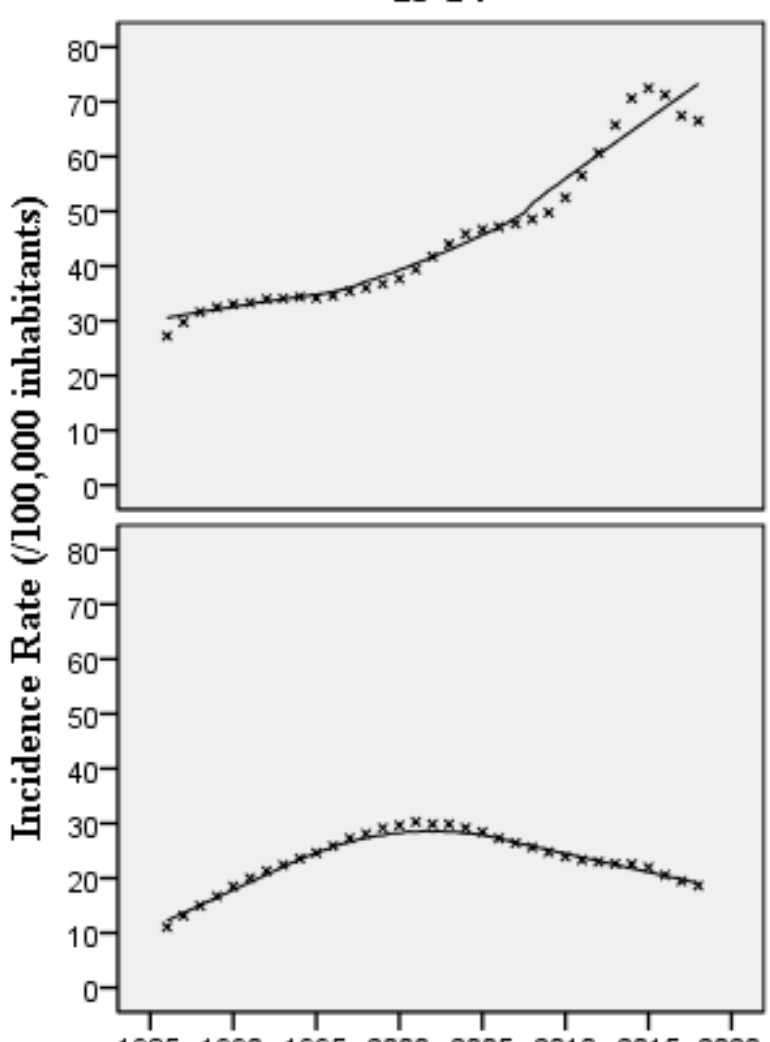

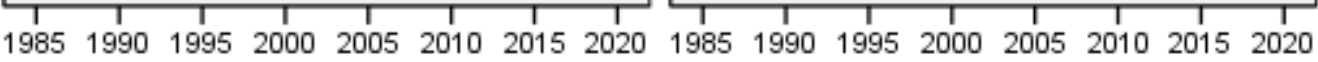

Year

Legend: $x$ Estimated Incidence Rate

Locally estimated scatterplot smoothing (LOESS)

\begin{tabular}{lcc}
\hline \multicolumn{3}{c}{ Coefficients of Determination $\left(\mathbf{R}^{2}\right)^{\mathbf{a}}$} \\
\hline Age group (years) & $\mathbf{M}$ & $\mathbf{F}$ \\
\hline $15-24$ & $0.95^{*}$ & $0.98^{*}$ \\
$\geq 25$ & $0.99^{*}$ & $0.99^{*}$ \\
\hline
\end{tabular}

" p-value $<0.001$.

${ }^{\mathrm{a}} R^{2}=\frac{\sum_{i}\left(\hat{y}_{i}-\bar{y}\right)^{2}}{\sum_{i}\left(y_{i}-\bar{y}\right)^{2}}, \hat{y}_{i}=$ LOESS values, $\bar{y}=$ mean of the observed values $y_{i}$.

FIGURE 1: HIV Incidence Rates (per 100,000 inhabitants) by sex and age group. Brazil. 1986-2018.

$5 / 11$ 
TABLE 2: Comparison of HIV incidence and HIV incidence rate estimates (per 100,000 inhabitants) with GBD estimates by sex and year of HIV infection. Brazil, 2000-2018.

\begin{tabular}{|c|c|c|c|c|c|c|c|c|c|c|c|c|}
\hline \multirow{4}{*}{ Year } & \multicolumn{12}{|c|}{ Males } \\
\hline & \multicolumn{6}{|c|}{ Brazilian estimates } & \multicolumn{6}{|c|}{ GBD estimates } \\
\hline & \multirow{2}{*}{ Incidence $(x 1000)$} & \multicolumn{2}{|c|}{$95 \% \mathrm{Cl}$} & \multirow{2}{*}{$\begin{array}{c}\text { Rate } \\
(/ 100000)\end{array}$} & \multicolumn{2}{|c|}{$95 \% \mathrm{Cl}$} & \multirow{2}{*}{$\begin{array}{c}\text { Incidence } \\
(\times 1000)\end{array}$} & \multicolumn{2}{|c|}{$95 \% \mathrm{Cl}$} & \multirow{2}{*}{$\begin{array}{c}\text { Rate } \\
(/ 100000)\end{array}$} & \multicolumn{2}{|c|}{$95 \% \mathrm{Cl}$} \\
\hline & & LL & UL & & LL & UL & & LL & UL & & LL & UL \\
\hline 2000 & 23.0 & 19.4 & 27.7 & 40.2 & 34.0 & 48.4 & 27.9 & 21.4 & 34.0 & 48.8 & 37.4 & 59.5 \\
\hline 2001 & 24.0 & 20.9 & 27.9 & 41.2 & 35.9 & 47.9 & 25.3 & 20.3 & 30.6 & 43.4 & 34.8 & 52.5 \\
\hline 2002 & 24.9 & 21.7 & 28.9 & 41.9 & 36.5 & 48.7 & 24.6 & 19.7 & 29.8 & 41.4 & 33.2 & 50.2 \\
\hline 2003 & 25.8 & 22.5 & 29.9 & 42.6 & 37.2 & 49.4 & 24.7 & 19.6 & 29.8 & 40.8 & 32.4 & 49.3 \\
\hline 2004 & 26.3 & 23.1 & 30.4 & 42.7 & 37.4 & 49.3 & 24.9 & 20.0 & 30.0 & 40.4 & 32.4 & 48.6 \\
\hline 2005 & 26.7 & 23.5 & 30.8 & 42.5 & 37.3 & 49.0 & 25.5 & 20.7 & 30.4 & 40.6 & 32.9 & 48.4 \\
\hline 2006 & 27.2 & 23.9 & 31.3 & 42.5 & 37.3 & 48.8 & 26.4 & 21.2 & 31.4 & 41.2 & 33.1 & 49.0 \\
\hline 2007 & 27.8 & 24.5 & 31.9 & 42.5 & 37.4 & 48.8 & 27.3 & 21.9 & 32.5 & 41.8 & 33.5 & 49.8 \\
\hline 2008 & 28.3 & 25.0 & 32.4 & 42.5 & 37.6 & 48.6 & 28.5 & 22.8 & 33.9 & 42.8 & 34.2 & 50.9 \\
\hline 2009 & 28.8 & 25.6 & 32.8 & 42.4 & 37.7 & 48.3 & 30.3 & 23.6 & 36.4 & 44.6 & 34.7 & 53.6 \\
\hline 2010 & 29.6 & 26.5 & 33.5 & 42.7 & 38.2 & 48.3 & 32.3 & 24.8 & 38.8 & 46.6 & 35.8 & 56.0 \\
\hline 2011 & 30.6 & 27.6 & 34.4 & 43.4 & 39.0 & 48.8 & 34.0 & 25.8 & 41.1 & 48.1 & 36.5 & 58.2 \\
\hline 2012 & 31.9 & 28.8 & 35.7 & 44.2 & 40.0 & 49.6 & 36.1 & 27.3 & 43.5 & 50.1 & 37.9 & 60.4 \\
\hline 2013 & 34.0 & 30.7 & 38.1 & 46.2 & 41.8 & 51.9 & 38.2 & 28.5 & 46.3 & 52.0 & 38.8 & 63.0 \\
\hline 2014 & 36.2 & 32.7 & 40.9 & 48.3 & 43.6 & 54.6 & 40.1 & 29.8 & 48.4 & 53.5 & 39.7 & 64.6 \\
\hline 2015 & 37.1 & 33.5 & 42.4 & 48.5 & 43.8 & 55.5 & 40.4 & 30.1 & 48.7 & 52.8 & 39.4 & 63.7 \\
\hline 2016 & 36.6 & 33.1 & 42.3 & 46.9 & 42.5 & 54.2 & 40.4 & 30.4 & 48.8 & 51.8 & 39.0 & 62.5 \\
\hline 2017 & 35.2 & 32.1 & 40.8 & 44.2 & 40.2 & 51.3 & 40.4 & 30.6 & 49.1 & 50.7 & 38.4 & 61.7 \\
\hline 2018 & 34.8 & 32.8 & 41.5 & 42.9 & 40.3 & 51.0 & 40.4 & 30.7 & 49.3 & 49.7 & 37.8 & 60.7 \\
\hline \multirow{4}{*}{ Year } & \multicolumn{12}{|c|}{ Females } \\
\hline & \multicolumn{6}{|c|}{ Brazilian estimates } & \multicolumn{6}{|c|}{ GBD estimates } \\
\hline & \multirow{2}{*}{ Incidence $(x 1000)$} & \multicolumn{2}{|c|}{$95 \% \mathrm{Cl}$} & \multirow{2}{*}{$\begin{array}{c}\text { Rate } \\
(/ 100000)\end{array}$} & \multicolumn{2}{|c|}{$95 \% \mathrm{Cl}$} & Incidence & & & & & \\
\hline & & LL & UL & & & & $(x 1000)$ & LL & UL & $(/ 100000)$ & LL & UL \\
\hline 2000 & 15.7 & 13.2 & 19.1 & 26.0 & 21.9 & 31.7 & 19.4 & 14.5 & 23.6 & 32.2 & 24.1 & 39.2 \\
\hline 2001 & 16.4 & 14.3 & 19.0 & 26.7 & 23.3 & 31.0 & 17.6 & 13.6 & 21.5 & 28.7 & 22.2 & 35.0 \\
\hline 2002 & 16.8 & 14.8 & 19.4 & 26.8 & 23.6 & 31.0 & 17.1 & 12.9 & 21.2 & 27.3 & 20.6 & 33.9 \\
\hline 2003 & 17.1 & 15.1 & 19.6 & 26.8 & 23.7 & 30.8 & 16.8 & 12.7 & 21.2 & 26.4 & 19.9 & 33.3 \\
\hline 2004 & 17.1 & 15.2 & 19.5 & 26.2 & 23.3 & 30.0 & 16.6 & 12.6 & 21.0 & 25.5 & 19.4 & 32.3 \\
\hline 2005 & 16.9 & 15.1 & 19.2 & 25.5 & 22.8 & 29.0 & 16.5 & 12.6 & 21.0 & 24.9 & 19.0 & 31.7 \\
\hline 2006 & 16.5 & 14.8 & 18.8 & 24.5 & 21.9 & 27.8 & 16.7 & 12.7 & 21.2 & 24.7 & 18.8 & 31.4 \\
\hline 2007 & 16.3 & 14.6 & 18.5 & 23.7 & 21.3 & 26.9 & 16.8 & 12.8 & 21.5 & 24.4 & 18.6 & 31.2 \\
\hline 2008 & 16.2 & 14.6 & 18.3 & 23.0 & 20.7 & 26.0 & 17.2 & 13.0 & 22.2 & 24.5 & 18.5 & 31.6 \\
\hline 2009 & 16.0 & 14.5 & 18.0 & 22.3 & 20.2 & 25.1 & 17.8 & 13.1 & 23.3 & 24.8 & 18.3 & 32.5 \\
\hline 2010 & 15.8 & 14.4 & 17.6 & 21.6 & 19.7 & 24.1 & 18.6 & 13.5 & 24.6 & 25.4 & 18.5 & 33.7 \\
\hline 2011 & 15.5 & 14.2 & 17.2 & 20.8 & 19.1 & 23.1 & 19.3 & 13.8 & 25.3 & 25.9 & 18.5 & 33.9 \\
\hline 2012 & 15.4 & 14.1 & 17.0 & 20.2 & 18.6 & 22.3 & 20.2 & 13.8 & 26.1 & 26.5 & 18.1 & 34.3 \\
\hline 2013 & 15.4 & 14.2 & 17.0 & 19.9 & 18.3 & 22.0 & 21.2 & 14.7 & 27.2 & 27.3 & 18.9 & 35.0 \\
\hline 2014 & 15.5 & 14.3 & 17.3 & 19.6 & 18.0 & 21.8 & 22.4 & 15.5 & 28.4 & 28.3 & 19.6 & 35.8 \\
\hline 2015 & 15.3 & 14.0 & 17.3 & 18.9 & 17.3 & 21.4 & 22.2 & 15.5 & 28.0 & 27.4 & 19.2 & 34.6 \\
\hline 2016 & 14.6 & 13.3 & 16.7 & 17.6 & 16.1 & 20.2 & 22.4 & 15.7 & 28.1 & 27.1 & 19.0 & 34.0 \\
\hline 2017 & 14.0 & 12.8 & 16.2 & 16.6 & 15.2 & 19.2 & 22.4 & 15.8 & 28.0 & 26.6 & 18.7 & 33.2 \\
\hline 2018 & 13.6 & 12.5 & 16.0 & 15.8 & 14.6 & 18.6 & 22.4 & 15.9 & 28.0 & 26.0 & 18.5 & 32.5 \\
\hline
\end{tabular}

Information sources: Brazilian estimates - Department of Chronic Conditions and Sexually Transmitted Diseases (DCCI), Ministry of Health. GBD estimates: https://vizhub.healthdata.org/gbd-compare/.

women, similar estimates were also found until 2009. However, GBD estimates show an upward trend from 2009 to 2014 and steadiness after year 2014, with approximately 22,000 new infections each year, while Brazilian estimates present a decreasing trend so that the differences widen at the end of the time series. Among women, the average ratio between estimates was 1.05 until 2009 but attains values higher than 1.50 in the last three years (Table 2).
In Figure 2, trends of Brazilian and GBD incidence rate estimates are compared. Among men aged 15 years or over, all Brazilian estimates are within the GBD 95\% CIs. Time trends are also similar, but a sharper decrease was found in the Brazilian estimates in recent years. Among women, Brazilian rates are within the GBD 95\% CIs until 2014. However, as trends in incidence rates diverge after that year, Brazilian estimates move away from the lower limits of the GBD CI. 
TABLE 2: Comparison of HIV incidence and HIV incidence rate estimates (per 100,000 inhabitants) with GBD estimates by sex and year of HIV infection. Brazil. 2000-2018 (continuation).

\begin{tabular}{|c|c|c|c|c|c|c|c|c|c|c|c|c|}
\hline \multirow{4}{*}{ Year } & \multicolumn{12}{|c|}{ Total } \\
\hline & \multicolumn{6}{|c|}{ Brazilian estimates } & \multicolumn{6}{|c|}{ GBD estimates } \\
\hline & \multirow{2}{*}{ Incidence $(x 1000)$} & \multicolumn{2}{|c|}{$95 \% \mathrm{Cl}$} & \multirow{2}{*}{$\begin{array}{c}\text { Rate } \\
(/ 100000)\end{array}$} & \multicolumn{2}{|c|}{$95 \% \mathrm{Cl}$} & \multirow{2}{*}{$\begin{array}{c}\text { Incidence } \\
(x 1000)\end{array}$} & \multicolumn{2}{|c|}{$95 \% \mathrm{Cl}$} & \multirow{2}{*}{$\begin{array}{c}\text { Rate } \\
(/ 100000)\end{array}$} & \multicolumn{2}{|c|}{$95 \% \mathrm{Cl}$} \\
\hline & & LL & UL & & LL & UL & & LL & UL & & LL & UL \\
\hline 2000 & 38.7 & 32.6 & 46.7 & 32.9 & 27.8 & 39.8 & 47.3 & 36.1 & 57.3 & 40.3 & 30.7 & 48.8 \\
\hline 2001 & 40.4 & 35.2 & 46.9 & 33.8 & 29.5 & 39.2 & 42.9 & 34.0 & 52.0 & 35.9 & 28.4 & 43.5 \\
\hline 2002 & 41.7 & 36.4 & 48.3 & 34.2 & 29.9 & 39.6 & 41.7 & 32.8 & 50.7 & 34.2 & 26.9 & 41.6 \\
\hline 2003 & 42.9 & 37.6 & 49.5 & 34.5 & 30.3 & 39.9 & 41.5 & 32.7 & 50.7 & 33.4 & 26.3 & 40.8 \\
\hline 2004 & 43.4 & 38.2 & 49.9 & 34.3 & 30.2 & 39.4 & 41.5 & 32.8 & 50.6 & 32.8 & 25.9 & 40.0 \\
\hline 2005 & 43.6 & 38.5 & 50.0 & 33.8 & 29.8 & 38.7 & 42.0 & 33.4 & 51.1 & 32.5 & 25.9 & 39.6 \\
\hline 2006 & 43.7 & 38.7 & 50.1 & 33.2 & 29.4 & 38.0 & 42.9 & 33.9 & 52.1 & 32.6 & 25.8 & 39.6 \\
\hline 2007 & 44.1 & 39.1 & 50.4 & 32.9 & 29.1 & 37.5 & 44.2 & 35.0 & 53.6 & 32.9 & 26.1 & 39.9 \\
\hline 2008 & 44.5 & 39.6 & 50.6 & 32.5 & 28.9 & 37.0 & 45.8 & 35.7 & 55.8 & 33.5 & 26.1 & 40.8 \\
\hline 2009 & 44.8 & 40.1 & 50.7 & 32.1 & 28.7 & 36.4 & 48.0 & 36.8 & 59.5 & 34.4 & 26.4 & 42.6 \\
\hline 2010 & 45.4 & 40.9 & 51.1 & 31.9 & 28.7 & 35.9 & 50.9 & 38.3 & 63.0 & 35.8 & 26.9 & 44.3 \\
\hline 2011 & 46.2 & 41.8 & 51.6 & 31.8 & 28.8 & 35.6 & 53.3 & 39.8 & 65.9 & 36.7 & 27.4 & 45.4 \\
\hline 2012 & 47.2 & 43.0 & 52.7 & 31.9 & 29.0 & 35.6 & 56.2 & 41.7 & 69.1 & 37.9 & 28.1 & 46.6 \\
\hline 2013 & 49.4 & 44.9 & 55.2 & 32.7 & 29.7 & 36.5 & 59.3 & 43.5 & 72.6 & 39.2 & 28.8 & 48.0 \\
\hline 2014 & 51.7 & 47.0 & 58.2 & 33.5 & 30.5 & 37.8 & 62.5 & 45.4 & 76.4 & 40.5 & 29.4 & 49.5 \\
\hline 2015 & 52.5 & 47.5 & 59.7 & 33.3 & 30.2 & 37.9 & 62.6 & 46.0 & 76.4 & 39.8 & 29.2 & 48.5 \\
\hline 2016 & 51.2 & 46.4 & 58.9 & 31.9 & 28.9 & 36.7 & 62.8 & 46.4 & 76.4 & 39.1 & 28.9 & 47.6 \\
\hline 2017 & 49.1 & 44.8 & 57.0 & 30.0 & 27.4 & 34.8 & 62.9 & 46.8 & 77.8 & 38.4 & 28.6 & 47.5 \\
\hline 2018 & 48.5 & 45.3 & 57.5 & 29.0 & 27.1 & 34.3 & 62.8 & 47.0 & 77.4 & 37.5 & 28.1 & 46.3 \\
\hline
\end{tabular}

Information sources: Brazilian estimates - Department of Chronic Conditions and Sexually Transmitted Diseases (DCCl), Ministry of Health. GBD estimates: https://vizhub.healthdata.org/gbd-compare/.

Progress indicators of epidemic control interventions are presented in Table 3. Annual growth rates of HIV incidence rates in the period 2000-2018 indicate that the highest annual growth was presented by the youngest age group (3.7\%). Men aged 25 years or over showed a small but significant decreasing rate $(-0.5 \%$ per year). Both female age groups showed decreasing rates of variation $(-2.7 \%$ and $-2.9 \%$ ), among women aged $15-24$ and 25 or over, respectively.

Improvements in the average time from infection to diagnosis were found for all age groups and both sexes (Table 3). Among men aged 15-24 years, the average time decreased from 9.0 to 3.9 years, and the estimated proportion of cases diagnosed less than one year after infection increased from $11.0 \%$ to $52.5 \%$ from 2000 to 2018 . Males aged 25 years or over presented the lowest average time from infection to diagnosis ( 2.8 years), and the highest proportion of people diagnosed in the same year of infection (60.6\%) in 2018. Among the youngest men who became HIV infected from 2000 to 2018 , the proportion of undiagnosed cases by 2018 was $22.6 \%$, and among the youngest women, $11.9 \%$, while among the oldest, the proportions were $13.4 \%$ among males and $12.9 \%$ among females. There is an increase in the proportion of HIV cases with antiretroviral dispensation within the first year of infection, reaching $48.1 \%$ among men and $42.6 \%$ among women in 2018 .

\section{DISCUSSION}

In this study, surveillance available data was used to estimate HIV incidence categorized by sex and age group in Brazil. The approach is applicable at granular levels to all countries that monitor the clinical information of patients (CD4 count and ART). The procedure used in this study represents a simplification when compared to the previous mode ${ }^{25}$ and uses the number of HIV cases

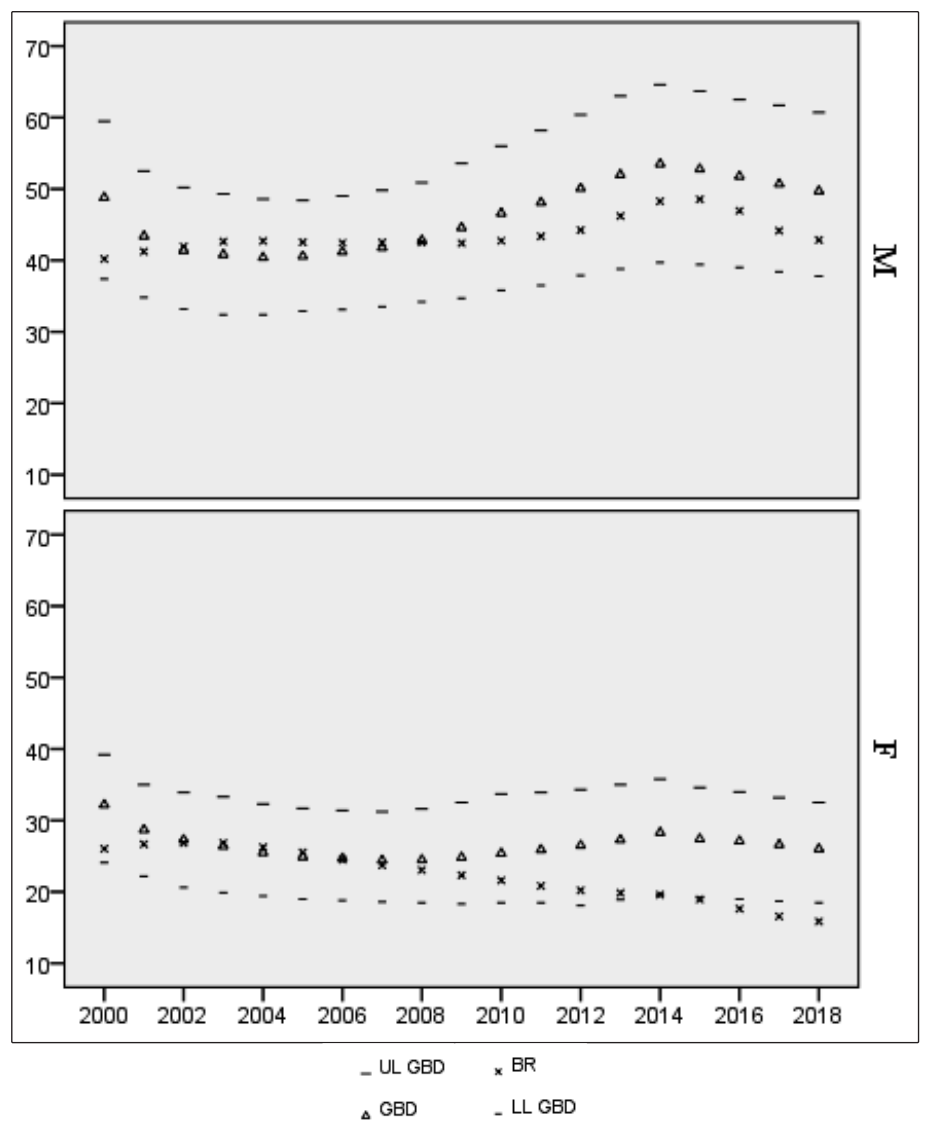

FIGURE 2: Brazilian and GBD estimates of HIV Incidence Rates (per 100,000 inhabitants) by sex. Brazil. 2000-2018. *GBD - GBD Incidence Rate (/100,000 inhabitants) estimate. UL GBD - 95\% CI Upper limit of the GBD estimate. LL GBD - 95\% CI Lower limit of the GBD estimate. BR - Brazilian Incidence Rate (/100,000 inhabitants). 
TABLE 3: Mean time from HIV infection to diagnosis. Proportion (\%) of HIV infected cases diagnosed within the first year of infection. Proportion (\%) with ART dispensation within the first year of HIV infection. Proportion of undiagnosed cases from 2010 to 2018 . HIV incidence rate annual growth ${ }^{\text {a from }} 2000$ to 2018 by sex and age group. Brazil. selected years.

\begin{tabular}{|c|c|c|c|c|c|c|c|}
\hline Sex & Age Group & $\begin{array}{l}\text { Year of HIV } \\
\text { infection }\end{array}$ & $\begin{array}{c}\text { Mean time } \\
\text { (years) }\end{array}$ & $\begin{array}{l}\text { Proportion (\%) } \\
\text { of diagnosed } \\
\text { cases in the } \\
\text { HIV infection } \\
\text { year }\end{array}$ & $\begin{array}{c}\text { Proportion } \\
\text { (\%) with ART } \\
\text { dispensation } \\
\text { in the HIV } \\
\text { infection year }\end{array}$ & $\begin{array}{l}\text { Proportion (\%) } \\
\text { of undiagnosed } \\
\text { cases from } \\
2000 \text { to } 2018\end{array}$ & $\begin{array}{l}\text { HIV incidence } \\
\text { rate annual } \\
\text { variation }{ }^{\mathrm{a}} \\
(\%)\end{array}$ \\
\hline \multirow{15}{*}{ Males } & \multirow{5}{*}{$15-24$} & 2000 & 9.0 & 11.5 & 0.5 & \multirow{5}{*}{22.6} & \multirow{5}{*}{$3.7^{*}$} \\
\hline & & 2005 & 8.6 & 11.6 & 0.2 & & \\
\hline & & 2010 & 6.1 & 20.9 & 1.0 & & \\
\hline & & 2014 & 5.0 & 35.2 & 14.7 & & \\
\hline & & 2018 & 3.9 & 52.5 & 45.0 & & \\
\hline & \multirow{5}{*}{$25+$} & 2000 & 5.0 & 26.9 & 3.8 & \multirow{5}{*}{13.4} & \multirow{5}{*}{$-0.5^{*}$} \\
\hline & & 2005 & 4.3 & 27.6 & 1.9 & & \\
\hline & & 2010 & 3.5 & 35.2 & 5.3 & & \\
\hline & & 2014 & 3.2 & 50.0 & 24.4 & & \\
\hline & & 2018 & 2.8 & 60.6 & 50.1 & & \\
\hline & \multirow{5}{*}{ Total } & 2000 & 6.5 & 22.7 & 2.4 & \multirow{5}{*}{16.3} & \multirow{5}{*}{$0.7^{*}$} \\
\hline & & 2005 & 5.6 & 22.8 & 1.2 & & \\
\hline & & 2010 & 4.8 & 30.7 & 3.6 & & \\
\hline & & 2014 & 3.7 & 44.6 & 20.6 & & \\
\hline & & 2018 & 3.4 & 57.8 & 48.1 & & \\
\hline \multirow{15}{*}{ Females } & \multirow{5}{*}{$15-24$} & 2000 & 5.9 & 23.8 & 2.2 & \multirow{5}{*}{11.9} & \multirow{5}{*}{$-2.7^{*}$} \\
\hline & & 2005 & 5.3 & 31.1 & 2.4 & & \\
\hline & & 2010 & 4.6 & 40.0 & 8.4 & & \\
\hline & & 2014 & 4.1 & 49.0 & 22.1 & & \\
\hline & & 2018 & 3.3 & 56.4 & 41.9 & & \\
\hline & \multirow{5}{*}{$25-+$} & 2000 & 3.5 & 28.8 & 4.2 & \multirow{5}{*}{12.9} & \multirow{5}{*}{$-2.9^{*}$} \\
\hline & & 2005 & 3.3 & 33.5 & 2.8 & & \\
\hline & & 2010 & 3.2 & 39.2 & 6.7 & & \\
\hline & & 2014 & 3.0 & 50.7 & 23.1 & & \\
\hline & & 2018 & 2.9 & 57.1 & 43.1 & & \\
\hline & \multirow{5}{*}{ Total } & 2000 & 5.3 & 27.2 & 3.3 & \multirow{5}{*}{12.6} & \multirow{5}{*}{$-2.8^{*}$} \\
\hline & & 2005 & 4.7 & 32.8 & 2.6 & & \\
\hline & & 2010 & 4.1 & 39.4 & 7.0 & & \\
\hline & & 2014 & 3.7 & 50.2 & 22.6 & & \\
\hline & & 2018 & 3.0 & 56.6 & 42.6 & & \\
\hline
\end{tabular}

${ }^{*}$ p-value less than 0.01 .

${ }^{a}$ Estimated by an exponential regression model assuming that incidence rates vary from 2000 to 2018 with a constant percent variation by year.

diagnosed within the first year of infection to generate estimates in recent years.

This method produced estimates consistent with those of the UNAIDS report $2019^{32}$. Like our results, estimates resulting from an age-structured deterministic model with reporting and treatment rates showed a second wave of infections after 2001 for both sexes, with the female curve decreasing after $2009^{33}$.

Regarding GBD estimates among men, HIV incidence rates similar, as are time trends from 2000 to 2018. Although all CIs overlap, small differences were found in recent years, as Brazilian incidence rates based on surveillance data has a sharper decline after 2015 than GBD estimates. Among women, similar estimates were also found up to 2009, but different trends were found after this year when Brazilian estimates move away from the lower limit of the GBD CIs.

Results of this study showed an increase in the proportion of people diagnosed within the first year of infection, a decrease in the average time from infection to diagnosis, and improvements in the proportion of cases starting antiretroviral therapy less than one year after HIV infection. The implementation of the strategy of treatment as prevention (TasP) in December 2013, early HIV diagnosis, and universal treatment at federal, state, and municipality levels, and other prevention interventions, such as pre-exposure prophylaxis (PreP), are having an impact on the control of the HIV epidemic in Brazil ${ }^{34,35}$. A recent study with patients on ART therapy in Brazil showed an increase in the proportion of cases with viral load suppression ${ }^{36}$. PrEP has also been implemented since December 2017 in Brazil ${ }^{37,38}$ and may also be reflected in these results. 
Decreases in undiagnosed HIV infection may well be attributable to intensified testing efforts in Brazil. These include multiple services for HIV testing, mobile health units, self-testing, and community-based rapid HIV testing strategies in partnership with non-governmental organizations (NGOs) ${ }^{39,40}$. Various national studies indicate that the proportion of people tested for HIV infection has increased, especially among most-at-risk population groups. Comparison of two rounds of biological behavioral surveillance surveys (BBSS) among men who have sex with men (MSM) showed the proportion of those who have never been tested for HIV decreased from 49.8\%, in 2009, to 33.8\%, in $2016^{41}$. Among female sex workers, relevant improvements were also found, with the proportion of HIV testing in the last 12 months increasing from $20.3 \%$ to $39.3 \%{ }^{42}$.

The continuous decrease in HIV incidence among young women may be attributed to government policy to reduce vertical transmission in Brazil ${ }^{43}$. Reduction in the number of HIV cases among children under five years of age and the proportion of HIV infected children among those exposed are clear indicators of the impact of the routine antenatal HIV screening and other care practices to prevent mother to child transmission ${ }^{44}$. In contrast, the group of youngest men (15-24 years of age) is the only group with a significant increase in the HIV incidence trend from 2000 to 2018. Furthermore, the highest percentage of undiagnosed incident cases from 2010 to 2018 was also found in this group.

One limitation of the Brazilian surveillance integrated system is the large proportion of missing data in the risk category, restricting HIV incidence analyses to available variables, such as age group, sex, and area of residence. However, the literature highlights that young men who has sex with men (MSM) have an increased risk of HIV infection, for which HIV infection rates are higher and are rising relative to the general population. Three nationwide studies of military recruits in 2002, 2007, and 2016 reported HIV prevalence among MSM of $0.56 \%, 1.23 \%$, and $1.32 \%$, respectively, while the overall recruit population prevalence remained stable, and much lower $(0.09 \%, 0.11 \% \text {, and } 0.12 \%)^{28,45}$. In a study conducted in two Brazilian cities using laboratory tests to distinguish recent from long-term infections, the estimated incidence rate was greater than $1 \%$ among MSM, more than 30 times greater than for heterosexual men in both cities ${ }^{19}$.

Other settings have experienced similar rises in incidence among $\mathrm{MSM}^{46,47}$. Despite earlier concerns about an increase in heterosexual cases and "feminization" of the epidemics in Brazil, it seems the actual scenario is the predominance of HIV infection among men, especially among the youngest age group. A recent online survey among MSM in Brazil revealed a low proportion of perceived HIV risk (26\%). Moreover, the younger age group increased the odds of binge drinking and unprotected receptive anal sex, and lowered odds of perceived HIV risk ${ }^{48}$. The lack of HIV risk perception is of concern because this may undermine control and prevention efforts, such as PreP. It can therefore be concluded that there is an urgent need to address the vulnerability of young MSM and identify interventions that could reshape notions and perceptions of risk in this group.
Furthermore, the present study indicates the need for expansion of public prevention policies focused on adolescents with more effective communication strategies, including the development of knowledge that involves motivation for a safer behavior ${ }^{49}$. Nevertheless, setbacks of religious and conservative positions have precluded sexual education activities in schools, which may explain the rising rates of unsafe sex among young people and the lack of risk perception, representing major challenges for public health policies aimed at controlling HIV epidemics in Brazil ${ }^{50}$.

Limitations of the Brazilian approach stem from possible violations of underlying CD4 model assumptions and from the imputation procedure used to estimate time from HIV infection to diagnosis. Additionally, as the estimation process relies on modelling the proportion of cases diagnosed within the first year of infection, estimates are uncertain in the more recent years, where we have less information about the expansion of early diagnosis, especially given the government policies of TasP and incentives to HIV testing. Although these limitations might influence the results, the method generated estimates consistent with that of other studies using different methodologies.

Updates to these estimates will enable professionals to monitor progress and plan effective interventions. Moreover, depending on the granularity of the data, the model can be used to derive estimates in other sub-populations and focus interventions on the most challenging population groups at subnational levels.

\section{ACKNOWLEDGMENTS}

We thank the institutions that provided support for the development and implementation of this study.

\section{FINANCIAL SUPPORT}

Agence National de Recherche sur le Sida et les Hépatites Virales - ANRS (process number 17154) and National Council for Scientific and Technological Development (process number 403883/2018-0).

\section{AUTHORS' CONTRIBUTION}

CLS participated in the design and writing of the article, data analysis, and discussion of results. PRBSJ, ARPP, RAC, RAR, GND participated in the methodology proposal, data analysis, and interpretation of results. DCM, MCP, and GFMP participated in the writing of the article and discussion of results. All authors approved the final version and are responsible for all aspects of the work in ensuring the accuracy and integrity of any part of the article.

\section{CONFLICT OF INTEREST}

No conflicting interests.

\section{ORCID}

Célia Landmann Szwarcwald: 0000-0002-7798-2095

Paulo Roberto Borges de Souza Júnior: 0000-0002-8142-4790

Ana Roberta Pati Pascom: 0000-0002-2646-3383 
Ronaldo de Almeida Coelho: 0000-0001-8667-0125

Rachel Abrahão Ribeiro: 0000-0001-9799-9233

Giseli Nogueira Damacena: 0000-0002-7059-3353

Deborah Carvalho Malta: 0000-0002-8214-5734

Maria Cristina Pimenta: 0000-0002-4205-9786

Gerson Fernandes Mendes Pereira: 0000-0001-8886-1662

\section{REFERENCES}

1. Van Sighem AI, Gras LA, Reiss P, Brinkman K, de Wolf F, ATHENA national observational cohort study. Life expectancy of recently diagnosed asymptomatic HIV-infected patients approaches that of uninfected individuals. AIDS. 2010;24(10):1527-35.

2. Stover J, Bollinger L, Izazola JA, Loures L, DeLay P, Ghys PD, et al. What Is Required to End the AIDS Epidemic as a Public Health Threat by 2030 ? The Cost and Impact of the Fast-Track Approach. PLoS One. 2016;11(5):e0154893.

3. Song R, Hall HI, Green TA, Szwarcwald CL, Pantazis N. Using CD4 Data to Estimate HIV Incidence, Prevalence, and Percent of Undiagnosed Infections in the United States. J Acquir Immune Defic Syndr. 2017;74(1):3-9.

4. Birrell PJ, Gill ON, Delpech VC, Brown AE, Desai S, Chadborn TR, et al. HIV incidence in men who have sex with men in England and Wales 2001-10: a nationwide population study. Lancet Infect Dis. 2013;13(4):313-8.

5. Rehle T, Shisana O, Pillay V, Zuma K, Puren A, Parker W. National HIV incidence measures-new insights into the South African epidemic. S Afr Med J. 2007;97(3):194-9.

6. Marty L, Cazein F, Panjo H, Pillonel J, Costagliola D, Supervie V, et al. Revealing geographical and population heterogeneity in HIV incidence, undiagnosed HIV prevalence and time to diagnosis to improve prevention and care: estimates for France. J Int AIDS Soc. 2018;21(3):e25100

7. Brookmeyer R. Measuring the HIV/AIDS epidemic: approaches and challenges. Epidemiol Rev. 2010;32:26-37.

8. Morgado MG, Bastos FI. Estimates of HIV-1 incidence based on serological methods: a brief methodological review. Cad Saude Publica. 2011;27(Suppl 1):S7-18.

9. Hallett TB, Zaba B, Stover J, Brown T, Slaymaker E, Gregson S, et al. Embracing different approaches to estimating HIV incidence, prevalence and mortality. AIDS. 2014;28(Suppl 4):S523-32.

10. Brookmeyer R, Gail MH. AIDS Epidemiology: A Quantitative Approach. Oxford University Press, New York; 1994. 372 p.

11. Montaner JS, Lima VD, Harrigan PR, Lourenço L, Yip B, Nosyk B. Expansion of HAART coverage is associated with sustained decreases in HIV/AIDS morbidity, mortality and HIV transmission: the "HIV Treatment as Prevention" experience in a Canadian setting. PLoS One. 2014;9:e87872.

12. Hallett TB, Zaba B, Todd J, Lopman B, Mwita W, Biraro S, et al. Estimating incidence from prevalence in generalised HIV epidemics: methods and validation. PLoS Med. 2008;5:e80.

13. Stover J, Brown T, Puckett R, Peerapatanapokin W. Updates to the Spectrum/ Estimations and Projections Package model for estimating trends and current values for key HIV indicators. AIDS. 2017;31:S5-11.
14. Janssen RS, Satten GA, Stramer SL, Rawal BD, O'Brien TR, Weiblen BJ, et al. New testing strategy to detect early HIV-1 infection for use in incidence estimates and for clinical and prevention purposes. JAMA. 1998;280(1):42-8.

15. Hall HI, Song R, Rhodes P, Prejean J, An Q, Lee LM, et al. Estimation of HIV incidence in the United States. JAMA. 2008;300(5):520-9.

16. Hu DJ, Vanichseni S, Mock PA, Young NL, Dobbs T, Byers RH Jr., et al. HIV type 1 incidence estimates by detection of recent infection from a cross-sectional sampling of injection drug users in Bangkok: use of the IgG capture BED enzyme immunoassay. AIDS Res Hum Retroviruses 2003;19(9):727-30.

17. Garrett $\mathrm{Nj}$, Lattimore S, Gilbart V, Aghaizu A, Mensah G, Tosswill J, et al. The Recent Infection Testing Algorithm (RITA) in clinical practice: a survey of HIV clinicians in England and Northern Ireland. HIV Med. 2012;13(7):444-7.

18. Zhu Q, Wang Y, Liu J, Duan X, Chen M, Yang J, et al. Identifying major drivers of incident HIV infection using recent infection testing algorithms (RITAs) to precisely inform targeted prevention. Int J Infect Dis. 2020;101:131-7.

19. Szwarcwald CL, Ferreira Oda C Júnior, Brito AM, Luhm KR, Ribeiro CE, Silva AM, et al. Estimation of HIV incidence in two Brazilian municipalities, 2013. Rev Saude Publica. 2016;50:55.

20. Rice BD, Elford J, Yin Z, Delpech VC. A new method to assign country of HIV infection among heterosexuals born abroad and diagnosed with HIV. AIDS. 2012;26(15):1961-6.

21. Ndawinz JD, Costagliola D, Supervie V. New method for estimating HIV incidence and time from infection to diagnosis using HIV surveillance data: results for France. AIDS. 2011;25(15):1905-13.

22. Szwarcwald CL, Pascom ARP, de Souza Júnior PR. Estimation of the HIV Incidence and of the Number of People Living With HIV/AIDS in Brazil, 2012. J AIDS Clin Res 2015;6:430.

23. Satcher Johnson A, Song R, Hall HI. Estimated HIV Incidence, Prevalence, and Undiagnosed Infections in US States and Washington, DC, 2010-2014. J Acquir Immune Defic Syndr. 2017;76(2):116-22.

24. Marty L, Van Beckhoven D, Ost C, Deblonde J, Costagliola D, Sasse A, et al. Estimates of the HIV undiagnosed population in Belgium reveals higher prevalence for MSM with foreign nationality and for geographic areas hosting big cities. J Int AIDS Soc. 2019;22(8):e25371.

25. Szwarcwald C, de Souza-Júnior PB, Pascom AR, Ferreira O. Results from a method for estimating HIV incidence based on the first CD4 count among treatment naïve cases: Brazil, 2004-2013. J AIDS Clin Res. 2016;7(627):2.

26. Lodi S, Phillips A, Touloumi G, Geskus R, Meyer L, Thiébaut R, et al. Time from human immunodeficiency virus seroconversion to reaching CD4+ cell count thresholds $<200,<350$, and $<500 \mathrm{Cells} / \mathrm{mm}^{3}$ : assessment of need following changes in treatment guidelines. Clin Infect Dis. 2011;53(8):817-25.

27. Crowell TA, Ganesan A, Berry SA, Deiss RG, Agan BK, Okulicz JF, et al. Hospitalizations among HIV controllers and persons with medically controlled HIV in the U.S. Military HIV Natural History Study. J Int AIDS Soc. 2016;19(1):20524.

28. Saffier IP, Kawa H, Harling G. A scoping review of prevalence, incidence and risk factors for HIV infection amongst young people in Brazil. BMC Infect Dis. 2017;17(1):675.

29. Sperhacke RD, da Motta LR, Kato SK, Vanni AC, Paganella MP, Oliveira MCP, et al. HIV prevalence and sexual behavior among young male conscripts in the Brazilian army, 2016. Medicine (Baltimore). 2018;97(1S Suppl 1):S25-31. 
30. Ministério da Saúde (MS). Secretaria de Vigilância em Saúde. Departamento de Vigilância, Prevenção e Controle das Infecções Sexualmente Transmissíveis, do HIV/Aids e das Hepatites Virais. Boletim Epidemiológico HIV/AIDS 2020. Brasília: MS; 2020. 66 p.

31. Murray CJL, Kyu HH, Frank TD, Carter A, Jahagirdar D, Biehl MH, et al. Global, regional, and national incidence, prevalence, and mortality of HIV, 1980-2017, and forecasts to 2030, for 195 countries and territories: a systematic analysis for the Global Burden of Diseases, Injuries, and Risk Factors Study 2017. Lancet HIV 2019;6:e831-59.

32. UNAIDS. UNAIDS DATA 2019 [Internet]. Citado em 08/03/2021. Disponível em: https://www.unaids.org/sites/default/files/media asset/2019-UNAIDS-data_en.pdf

33. Mangal TD, Pascom ARP, Vesga JF, Meireles MV, Benzaken AS, Hallett TB. Estimating HIV incidence from surveillance data indicates a second wave of infections in Brazil. Epidemics. 2019;27:77-85.

34. Montaner JS, Lima VD, Harrigan PR, Lourenço L, Yip B, Nosyk B, et al. Expansion of HAART coverage is associated with sustained decreases in HIV/AIDS morbidity, mortality and HIV transmission: The "HIV Treatment as Prevention" experience in a Canadian setting. PLoS One. 2014;9:e87872.

35. Grinsztejn B, Hosseinipour MC, Ribaudo HJ, Swindells S, Eron J, Chen $Y Q$, et al. Effects of early versus delayed initiation of antiretroviral treatment on clinical outcomes of HIV-1 infection: Results from the phase 3 HPTN 052 randomised controlled trial. Lancet Infect Dis. 2014;14(4):281-90.

36. Pascom ARP, Meireles MV, Benzaken AS. Sociodemographic determinants of attrition in the HIV continuum of care in Brazil, in 2016. Medicine (Baltimore). 2018;97(1S Suppl 1):S69-74.

37. Sousa LRM, Elias HC, Fernandes NM, Gir E, Reis RK. Knowledge of PEP and PrEP among people living with HIV/aids in Brazil. BMC Public Health. 2021;21(1):64.

38. Marins LMS, Torres TS, Leite IDC, Moreira RI, Luz PM, Hoagland B, et al. Performance of HIV pre-exposure prophylaxis indirect adherence measures among men who have sex with men and transgender women: Results from the PrEP Brasil Study. PLoS One. 2019;14(8):e0221281.

39. Pascom ARP, de Barros CHD, Lobo TDM, Pasini EM, Comparini RA, de Mesquita FC. Point-of-care HIV tests done by peers, Brazil. Bulletin of the World Health Organization 2016;94:626-30.

40. de Barros CHD, Sabido M, Lobo TDM, Pascom ARP, Pasini E, de Mesquita FC, et al. Community-Based Rapid HIV Testing in Brazil for Vulnerable Populations: Whom are We Reaching? J AIDS Clin Res. 2017;8:1-7.
41. Guimarães MDC, Kendall C, Magno L, Rocha GM, Knauth DR, Leal $\mathrm{AF}$, et al. Comparing HIV risk-related behaviors between 2 RDS national samples of MSM in Brazil, 2009 and 2016. Medicine (Baltimore). 2018;97(1S Suppl 1):S62-68.

42. Szwarcwald CL, de Almeida WDS, Damacena GN, de Souza-Júnior PRB, Ferreira-Júnior ODC, Guimarães MDC, et al. Changes in attitudes, risky practices, and HIV and syphilis prevalence among female sex workers in Brazil from 2009 to 2016. Medicine (Baltimore). 2018;97(1S Suppl 1):S46-53.

43. Domingues RMSM, Saraceni V, Leal MDC. Mother to child transmission of HIV in Brazil: Data from the "Birth in Brazil study", a national hospital-based study. PLoS One. 2018;13(2):e0192985.

44. Ministério da Saúde (MS). Secretaria de Vigilância em Saúde. Departamento de Vigilância, Prevenção e Controle das Infecções Sexualmente Transmissíveis, do HIV/Aids e das Hepatites Virais. Relatório de monitoramento clínico do HIV 2019. Brasília: MS; 2019. $123 \mathrm{p}$.

45. Szwarcwald CL, Andrade CL, Pascom AR, Fazito E, Pereira GF, Penha IT. HIV-related risky practices among Brazilian young men, 2007. Cad Saude Publica. 2011;27(Suppl 1):S19-26.

46. van Sighem A, Vidondo B, Glass TR, Bucher HC, Vernazza P, Gebhardt $\mathrm{M}$, et al. Resurgence of HIV infection among men who have sex with men in Switzerland: mathematical modelling study. PLoS One. 2012;7(9):e44819.

47. Beyrer C, Sullivan P, Sanchez J, Baral SD, Collins C, Wirtz AL, et al. The increase in global HIV epidemics in MSM. AIDS. 2013;27:2665-78.

48. Luz PM, Torres TS, Almeida-Brasil CC, Marins LMS, Veloso VG, Grinsztejn B, et al. High-Risk Sexual Behavior, Binge Drinking and Use of Stimulants are Key Experiences on the Pathway to High Perceived HIV Risk Among Men Who Have Sex with Men in Brazil. AIDS Behav. 2021;25(3):748-57.

49. Guimarães MDC, Magno L, Ceccato MDGB, Gomes RRFM, Leal AF, Knauth DR, Veras MASM, Dourado I, Brito AM, Kendall C, Kerr LRFS; Brazilian HIV/MSM Surveillance Group. HIV/AIDS knowledge among MSM in Brazil: a challenge for public policies. Rev Bras Epidemiol. 2019;22(Suppl 1):e190005.

50. Felisbino-Mendes MS, Paula TF, Machado ÍE, Oliveira-Campos M, Malta DC. Analysis of sexual and reproductive health indicators of Brazilian adolescents, 2009, 2012 and 2015. Rev Bras Epidemiol. 2018;21(suppl 1). 